\title{
Biorefinery lignosulfonates as a dispersant for coal water slurry
}

\author{
Yanlin Qin ${ }^{1,2 \dagger}$, Dongjie Yang ${ }^{1 \dagger}$, Feng Gu ${ }^{2,3}$, Xuzhao $\mathrm{Li}^{1}$, Wenlong Xiong ${ }^{1}$ and J. Y. Zhu ${ }^{2 *}$
}

\begin{abstract}
Background: Valorization of lignin from biofuel production is the key to developing biorefinery technologies for sustainable and economic utilization of lignocellulosic biomass. Here we present isolating lignosulfonate from the spent liquors of Sulfite Pretreatment to Overcome the Recalcitrance of Lignocelluloses (SPORL)-pretreated lodgepole pine and Douglas-fir forest residue as a dispersant for coal water slurry. The two SPORL pretreatments were conducted at a pilot scale and resulted in very high ethanol yield from the pretreated biomass. Therefore, demonstrating the commercial utility of these lignosulfonates has practical significance.

Main results: The two isolated biorefinery lignosulfonates (LSs), Na-LS and Ca-LS, both had a molecular weight of approximately $9000 \mathrm{Da}$. Fundamental lignin properties such as chemical structure, functional groups were analyzed. The two LSs showed slightly better to equal performance in modifying CWS rheology than a commercial dispersant naphthalene sulfonate formaldehyde condensate (FDN), despite they were less sulfonated than FDN.

Conclusions: The practical importance of this study is that the pilot-scale pretreatments that produced the two LSs also produced excellent bioethanol yields at high titer without detoxification and washing. This suggests SPORL pretreatment is a promising technology for economic bioconversion of under-utilized woody biomass.
\end{abstract}

Keywords: Sodium and calcium lignosulfonate, Coal-water slurry, Viscosity, Dispersant, Adsorption, Lignin valorization

\section{Background}

The concept of biorefinery is to mimic petroleum refinery to produce multi-products such fuels, chemicals, polymers from a lignocellulosic feedstock to diversify product portfolio, avoid market saturation, and maximize resource utilization. The sugar platform as a major lignocellulosic biomass conversion pathway relies on the conversion of carbohydrates to sugars for subsequent processing to fuels and chemicals. While it is very attractive because sugars are flexible building blocks for producing a variety of chemicals and products [1], valorization of the lignin fraction is the key to commercial success because lignin is the second most abundant fraction in lignocelluloses of approximately 15-30 \% [2]. Current technologies for the sugar platform rely on a pretreatment (or

\footnotetext{
*Correspondence: jzhu@fs.fed.us

${ }^{\dagger}$ Yanlin Qin and Dongjie Yang contributed equally to this work

2 USDA Forest Service, Forest Products Laboratory, Madison, WI, USA

Full list of author information is available at the end of the article
}

fractionation) step followed by enzymatic saccharification of the pretreated solids [3]. Depending on the pretreatment process employed, lignin is often fractionated into a soluble fraction in the pretreatment spent liquor and a fraction retained in solids. The current utilization of these two lignin fractions-biorefinery lignin-remains as a low value boiler fuel as practiced in pulp mills, despite substantial research and development efforts have been made in bioconversion of lignocelluloses [4].

Here, we demonstrate a biorefinery lignin, i.e., the water soluble lignin fraction from Sulfite Pretreatment to Overcome the Recalcitrance of Lignocelluloses (SPORL) [5] of softwoods-lignosulfonate (LS), as a dispersant of coal water slurry (CWS) without further processing. Coal is an important energy source. Approximately $39 \%$ of the electricity was produced from coal in the U.S. (US Energy Information Administration). CWS was developed in the 1920s in Russia. Due to the shortage of oil supply in the 70s, CWS technologies was further developed as an 
alternative to liquid fuel in a variety of applications. CWS is a clean technology compared with coal itself which can alleviate many concerns of coal combustion $[6,7]$. For example, it can produce high combustion efficiency, low discharge of ash, and lower NOx and SOx air emissions $[8,9]$. Typical CWS contains $60-75 \%$ small suspended coal particles in $25-40 \%$ water, and $1 \%$ chemical dispersants. CWS can be directly burned without dewatering [10]. Dispersants play an important role to reduce CWS viscosity and stabilize rheological properties for good atomization and efficient combustion [11, 12]. To meet the potential demands for CWS, several dispersants such as naphthalene sulfonate formaldehyde condensate [13], sulfonated acetone-formaldehyde [14], carboxylate type copolymer [15], cardanol formaldehyde sulfonate [7], sodium polystyrene sulfonate [16], sodium dodecyl benzenesulfonate [17] have been studied. However, lignin based dispersants attracted great attention $[18,19]$.

The practical significance of this study is the existence of a mature commercial market for CWS dispersant and the valorization of LS from wood biorefinery as a coproduct. Furthermore, with the gradual closing of sulfite pulp mills in the last 40 years around the world, there is a shortage of commercial LS products. Some regions rely on a low quality LS derived from sulfonation of kraft lignin-from kraft pulping [20] to meet market demand. Therefore, LS from SPORL can be a commercially and economically viable co-product for biorefinery.

\section{Results and discussion}

\section{FT-IR and ${ }^{1} \mathrm{H}$-NMR spectra of LSs}

The FTIR spectra of the two biorefinery LSs, Na-LS and Ca-LS are shown in Fig. 1a and the band assignments are given in Table 1.

The band at $3420 \mathrm{~cm}^{-1}$ relates to the aromatic and aliphatic $\mathrm{OH}$ groups in lignin [21]. The peak at $1421 \mathrm{~cm}^{-1}$ confirms the presence of COO-group [22]. The bands at 1190 and $1037 \mathrm{~cm}^{-1}$ are from asymmetric and $\mathrm{S}=\mathrm{O}$ stretching vibration of $\mathrm{SO}_{3}^{2-}$ [23], respectively.

The two LSs were also analyzed by ${ }^{1} \mathrm{H}$-NMR spectroscopy (Fig. 1b). Chemical shift assignments are listed in Table 1. The regions of the 7.52-6.80 and 6.80-6.50 ppm are detected in the aromatic proton of the guaiacyl units and syringyl units [24], respectively. The signals at 6.00-4.00 ppm are $\mathrm{H}_{\alpha}, \mathrm{H}_{\beta}$ and $\mathrm{H}_{\gamma}$ in $\beta-\mathrm{O}-4^{\prime}, \beta-5^{\prime}$ and $\beta-\beta^{\prime}$ structure [25]. The signals between 3.32 and $3.10 \mathrm{ppm}$ correspond to $\mathrm{H}$ in phenolic hydroxyl group [24]. The signals at $2.3-2.1$ and $2.1-1.8 \mathrm{ppm}$ are owing to aromatic and aliphatic acetates [26], respectively.

\section{Dispersant molecular weight and function groups}

The functional group contents and molecular weight of a dispersant have great effects on its dispersion
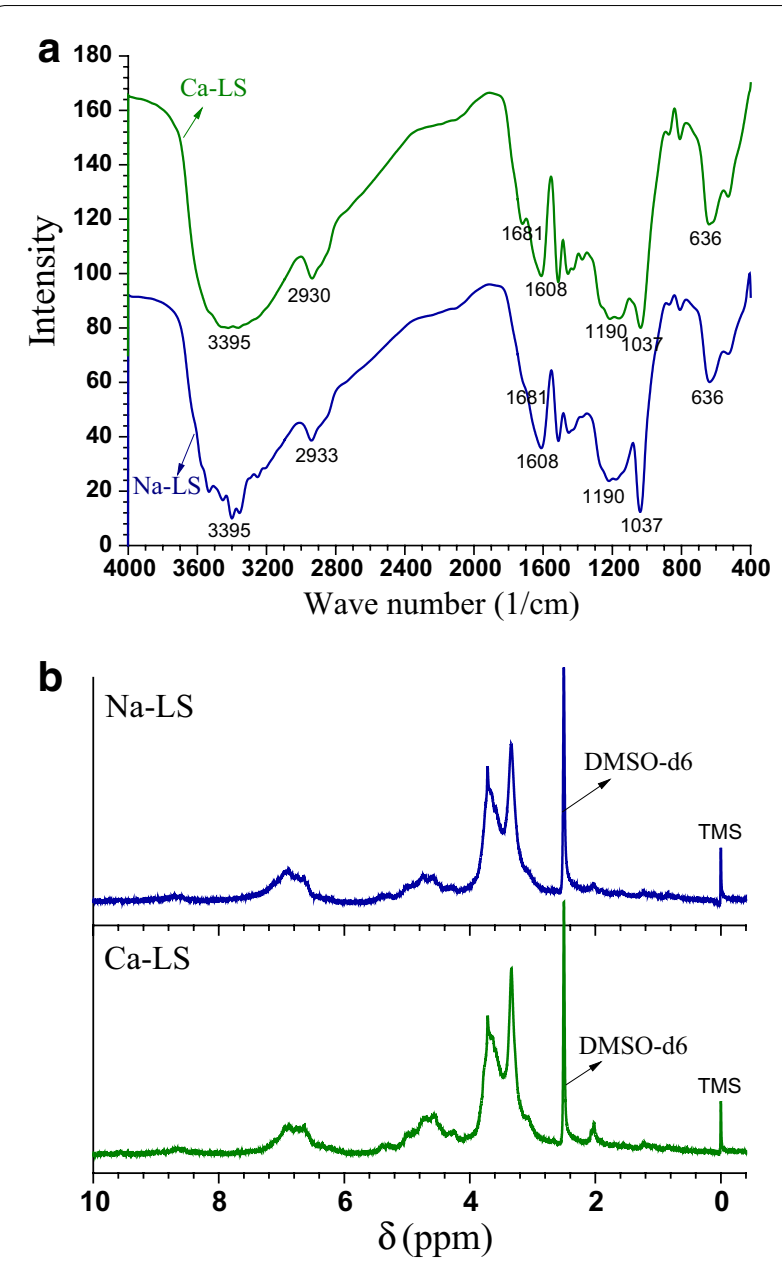

Fig. 1 a FTIR spectra of Na-LS and Ca-LS; $\boldsymbol{b}^{1} H$ NMR spectra of Na-LS and Ca-LS in DMSO-d

performance [10]. The molecular weight distributions of the two biorefinery LSs, Na-LS and Ca-LS and FDN were not substantially different with a peak at approximately 10, $000 \mathrm{Da}$ (Fig. 2; Table 2) especially when errors in calibration and measurements were taken into consideration. Both LSs had a slightly broader distribution.

FDN had much higher sulfonic acid group content than the two biorefinery LSs, and almost two times of that of Ca-LS (Table 2). However, the two biorefinery LSs also contained phenolic hydroxyl and carboxyl groups. Though Na-LS and Ca-LS were not substantially different, Na-LS was slightly more sulfonated with slightly higher phenolic hydroxyl content and lower carboxyl group content, in agreement with FTIR (Fig. 1a) and ${ }^{1} \mathrm{H}-$ NMR (Fig. 1b) analyses. The uncertainty analysis based on measured quantities in Eq. (1) showed a relative error of propagation of $2 \%$ while the measured relative errors reported in Table 2 were $2-6 \%$. 
Table 1 Assignments of lignin IR and ${ }^{1} H-N M R$ spectral bands

\begin{tabular}{ll}
\hline & Assignment \\
\hline IR wavenumber $\left(\mathrm{cm}^{-1}\right)$ & \\
3390 & $\mathrm{OH}$ stretching in phenolic and aliphatic structures \\
$2933 / 2930$ & $\mathrm{C}-\mathrm{H}$ vibration in $-\mathrm{CH}_{3}$ and $-\mathrm{CH}_{2}-$ \\
2850 & $\mathrm{C}-\mathrm{H}$ vibration in $\mathrm{CH}_{3} \mathrm{O}-$ \\
1681 & Conjugated carbonyl groups \\
1608 & Aromatic skeleton expansion vibration \\
1421 & $\mathrm{COO}-$ vibration \\
1190 & Asymmetric stretching vibration of $\mathrm{SO}_{3}^{2-}$ \\
1037 & Assignment $\mathrm{S}=\mathrm{O}$ stretching vibration \\
636 & $\mathrm{C}-\mathrm{O}-\mathrm{C}$ stretch stretching vibration \\
${ }^{1} \mathrm{H}-\mathrm{NMR}$ chemical shifts $(\mathrm{ppm})$ \\
$8.50-9.70$ & $\mathrm{H}$ in carboxylic acid \\
$7.25-6.80$ & The aromatic proton of the guaiacyl units \\
$6.80-6.50$ & aromatic protons of syringyl units \\
$5.50-4.00$ & $\mathrm{H}_{\alpha^{\prime}} \mathrm{H}_{\beta^{\prime}} \mathrm{H}_{\gamma}$ in $\beta$-O-4', $\beta-5$ ' and $\beta$ - $\beta^{\prime}$ 'structure \\
$4.00-3.32$ & $\mathrm{H}$ in methoxyls \\
$3.52-3.10$ & $\mathrm{H}$ in phenolic hydroxyl group \\
$2.7-2.3$ & DMSO \\
$2.3-2.1$ & $\mathrm{H}$ in aromatic acetates \\
$2.1-1.8$ & $\mathrm{H}$ in aliphatic acetates \\
&
\end{tabular}

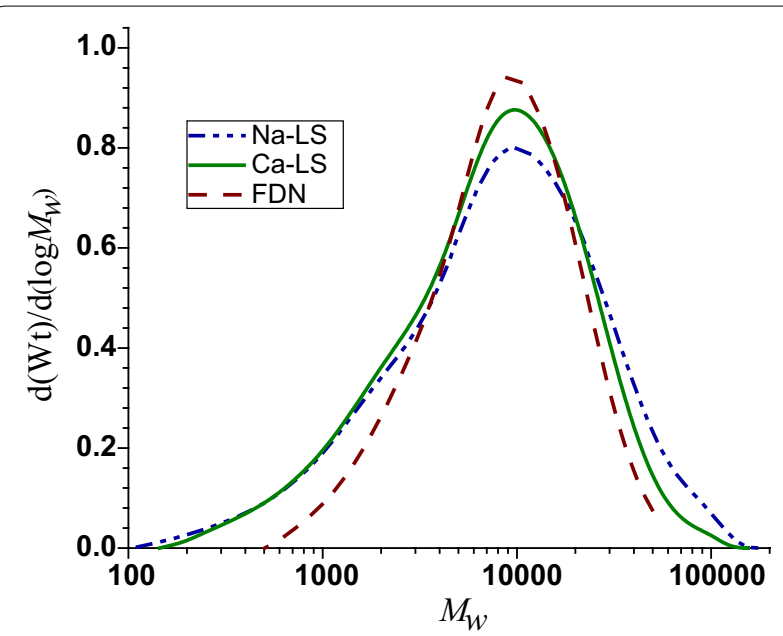

Fig. 2 The molecular weight distribution of the three CWS dispersants: Na-LS, Ca-LS, and FDN

\section{Adsorption of dispersants by coal particles}

The adsorption of dispersants onto coal particle surface is essential for dispersant to function in modifying CWS rheological properties to prevent flocculation and agglomeration. The adsorption isotherms of the two biorefinery LSs were similar to that of FDN (Fig. 3). The measurements errors in adsorption based on replicate measurements were small compared with adsorption variations among different samples using different dispersants. The Langmuir model was found not suitable for the non-homogeneous CWS system. However, the Freundlich model was not able to provide good fit of the adsorption data either despite it was designed for non-ideal and heterogeneous adsorption processes. The absorption data were therefore fitted using the RedlichPeterson model Eq. (1) [27, 28].

$$
Q=\frac{A Q_{f}}{1+B\left(Q_{f}\right)^{N}}
$$

where $Q$ is the amount of adsorbed (or bound) dispersant in $\mathrm{mg} / \mathrm{L}, Q_{f}$ is the free dispersant concentration in CWS in $\mathrm{mg} / \mathrm{L}, A$ and $B$ in $(\mathrm{L} / \mathrm{mg})^{1 / \mathrm{N}}$ are the Redlich-Peterson isotherm constants (Table 3), respectively. $\mathrm{N}$ is the exponential parameter.

The two Redlich-Peterson isotherm constants $A$ and $B$ were in the similar ranges for Ca-LS and FDN when fitting errors were taken into consideration (Table 3). This can be clearly seen form Fig. 3. However, Na-LS showed slightly more absorption than FDN despite FDN had a higher sulfonic acid group content (Table 2).

\section{Zeta potential of coal particles in CWS}

The adsorption between dispersant and coal particle surface was mainly through hydrogen bonding, electrostatic, and hydrophobic interactions [29]. Functional groups such as hydroxyl, carbonyl, carboxyl and methoxy groups existed on coal particle surface. In CWS system, coal particles can form double electrostatic layer due to the ionization of absorbing dispersant [10]. Hydroxyl, carbonyl and sulfonic acid groups of dispersants can also interact with coal particles and affect CWS dispersion. Zeta potential was used to characterize interfacial electrostatic interactions. The zeta potential of coal particles without the application of dispersant was approximately $-3.50 \mathrm{mV}$ (Fig. 4), indicating coal was minimally negatively charged at surface. Carboxylic and phenolic hydroxyl groups on the coal particle surface can improve ionization in solution [19], which resulted in increased (negatively) coal surface charge and can reduce the absorption of anionic dispersant onto coal surface. With the application of dispersant, zeta potential (absolute value) was increased continuously (Fig. 4). Applications of the biorefinery LSs, Na-LS and Ca-LS, resulted in slightly higher zeta-potential, i.e., more negatively charged coal, than using FDN, which can facilitate CWS dispersion. It is noticed that FDN had almost twice the amount of sulfonic acid groups content than the two biorefinery LSs (Table 2); however, the application of FDN resulted in a lower coal Zeta-potential (in absolute value) than that from the application of each LS. This 
Table 2 Functional group contents and molecular weights of three CWS dispersants

\begin{tabular}{|c|c|c|c|c|c|c|}
\hline \multirow[t]{2}{*}{ Sample } & \multicolumn{3}{|c|}{ Functional group content $(\mathrm{mmol} / \mathrm{g})$} & \multicolumn{3}{|c|}{ Molecular weight } \\
\hline & Sulfonic & Phenolic hydroxyl & Carboxyl & $M_{\mathrm{w}}$ & $M_{\mathrm{n}}$ & $M_{\mathrm{w}} / M_{\mathrm{n}}$ \\
\hline $\mathrm{Na}-\mathrm{LS}$ & $1.44 \pm 0.06$ & $1.84 \pm 0.07$ & $2.31 \pm 0.10$ & $9300 \pm 104$ & $7735 \pm 85$ & $1.20 \pm 0.03$ \\
\hline Ca-LS & $1.19 \pm 0.07$ & $1.65 \pm 0.08$ & $2.55 \pm 0.09$ & $8870 \pm 123$ & $7625 \pm 64$ & $1.17 \pm 0.04$ \\
\hline FDN & $2.24 \pm 0.04$ & 0 & 0 & $8100 \pm 76$ & $7700 \pm 46$ & $1.05 \pm 0.03$ \\
\hline
\end{tabular}

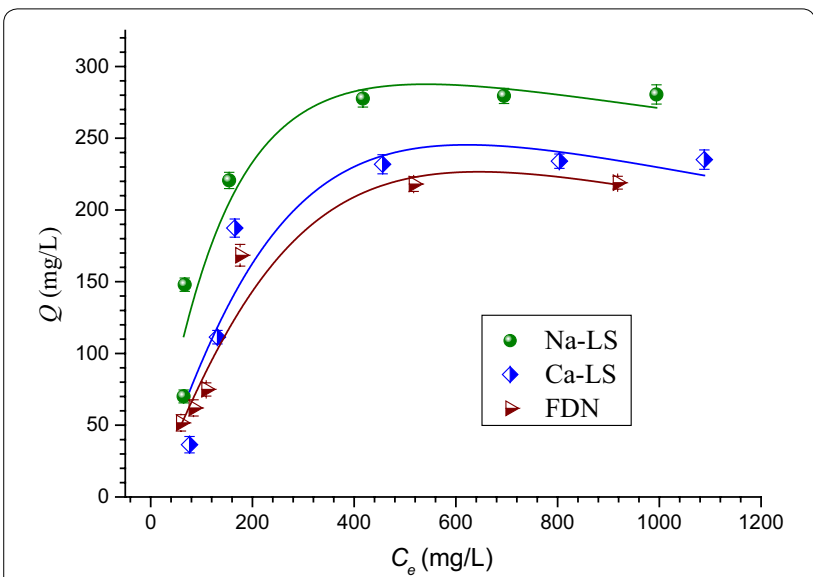

Fig. 3 Adsorption isotherms of CWS dispersants

Table 3 Parameters for predicting dispersant adsorption (at $1.0 \mathrm{wt} \%$ dosage) by coal using the Redlich-Peterson model

\begin{tabular}{lllll}
\hline & $\boldsymbol{A}(\mathbf{L} / \mathbf{g})$ & $\boldsymbol{B} \times \mathbf{1 \mathbf { 1 } ^ { \mathbf { 5 } }} \mathbf{( \mathbf { 1 } / \mathbf { m g } )}$ & $\boldsymbol{N}$ & $\boldsymbol{r}^{\mathbf{2}}$ \\
\hline Na-LS & $2.068 \pm 0.657$ & $67.2 \pm 17.3$ & $1.330 \pm 0.343$ & 0.859 \\
Ca-LS & $1.061 \pm 0.341$ & $6.21 \pm 2.87$ & $1.587 \pm 0.639$ & 0.820 \\
FDN & $0.934 \pm 0.187$ & $2.76 \pm 1.13$ & $1.699 \pm 0.592$ & 0.919 \\
\hline
\end{tabular}

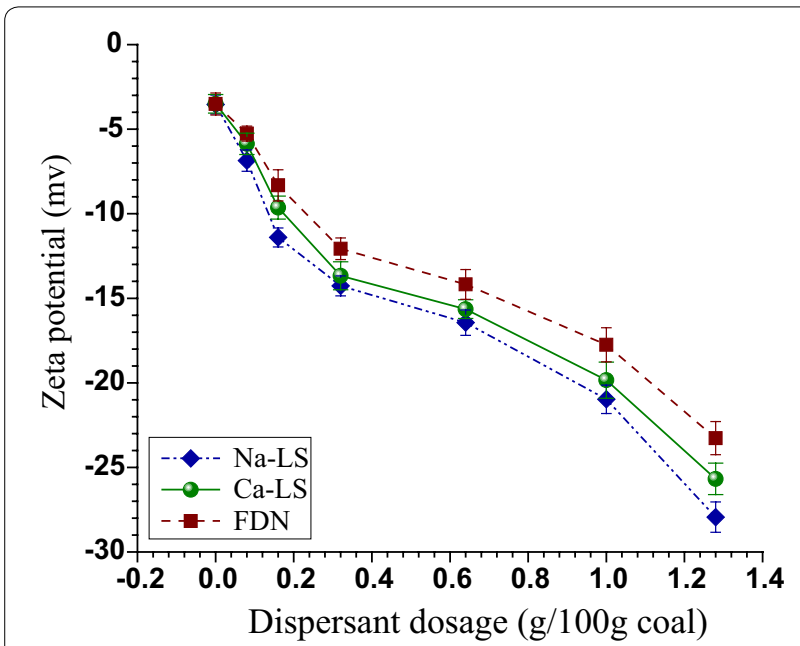

Fig. 4 Zeta potential of coal particles in CWS with the applciation of $\mathrm{Na}-\mathrm{LS}, \mathrm{Ca}-\mathrm{LS}$ and FDN indicated that the carboxyl and phenol-hydroxyl groups in LS also play an important role in dispersing CWS.

\section{Viscosity-reducing capacity of dispersants}

The CWS prepared in this study was very viscous and did not flow under normal rheological testing conditions without the application of a dispersant. At application dosages of 0.75 and $1.0 \mathrm{wt} \%$, similar performance in terms of modifying rheological properties of CWS was achieved when applying the two biorefinery LSs compared with the performance achieved using FDN (Fig. 5). The apparent viscosity of CWS decreased rapidly with increasing shear rate, i.e., shear thinning behavior. Replicate rheological tests at shear rate $100(1 / \mathrm{s})$ indicated that the standard deviations in apparent viscosity measurements were very low of less than $1 \%$, i.e., $\eta_{100}=624 \pm 5.9,698 \pm 6.7,857 \pm 6.5$ (mPa.s) for the CWS applied Na-LS, Ca-LS, FDN, respectively, suggesting the differences in modifying CWS viscosity by the three dispersants shown in Fig. 5 were significant. CWS applied with Na-LS resulted in the lowest shear stress, suggesting Na-LS performed better than FDN for CWS to disperse and flow. It is generally believed that divalent Ca-LS is not a suitable dispersant for CWS due to the destruction of the double electrostatic layer. However, the results showed that the performance of Ca-LS is equivalent to Na-LS, perhaps due to the low amount of $\mathrm{Ca}$. The stress-shear rate curves at dispersant dosage of $1.0 \mathrm{wt} \%$ suggested the CWSs dispersed by Na-LS and Ca-LS were Newtonian like. However, the CWS dispersed using FDN had two viscosities which was also observed in a previous study [30].

\section{Conclusions}

High value utilization of biorefinery lignin with minimal processing is critical to improve the commercial viability of biofuel production. This study demonstrated two biorefinery LSs directly isolated from the spent liquors of SPORL pretreatment of softwoods as dispersant for coal water slurry. Both biorefinery LSs showed slightly better or equal performance in modifying the rheological properties of CWS compared with a commercial dispersant FDN. Since, the SPORL conditions under which the two biorefinery LSs produced also prodcued excellent sugar 


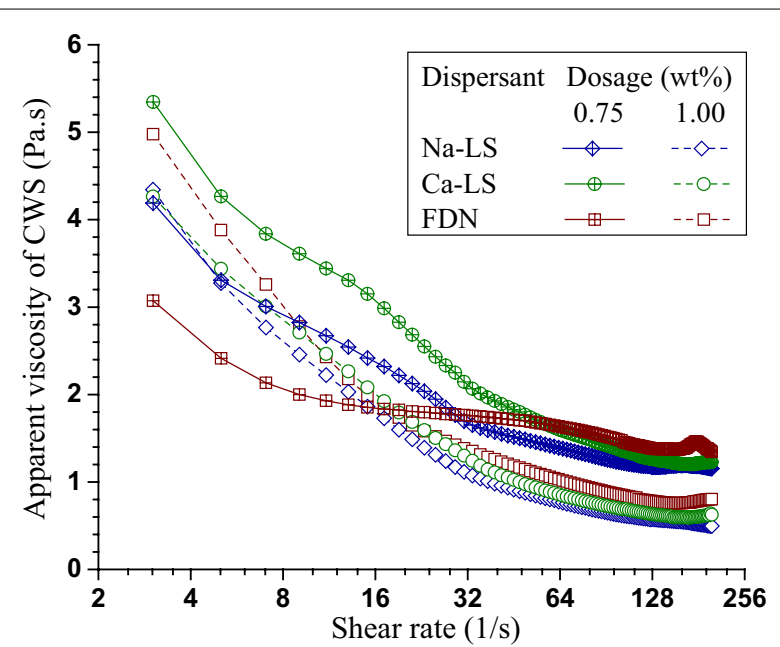

Fig. 5 Apparent viscosity and shear rate dependence curves of CWS with the application of dispersants at $1.0 \mathrm{wt} \%$

and biofuel yields at high titer without detoxification and solids washing, this study further supported the commercial viability of SPORL.

\section{Methods}

\section{Materials}

Two biorefinery LSs, Na-LS and Ca-LS, were separated from the spent liquors of SPORL pretreated mountain pine beetle killed lodgepole pine and Douglas-fir forest residue at a pilot-scale, respectively. These two feedstocks represent low grade woody biomass with limited or no value for lumber or fiber production. The pretreatment conditions for these two feedstocks along with ethanol yields from the subsequent enzymatic saccharification and fermentation of the pretreated whole slurries were listed in Table 4. Detailed descriptions of the two pretreatments and ethanol production can be found in the previously studies [31,32]. After each pretreatment, the woody materials remained intact, therefore, the spent liquor can be easily separately as freely drainable liquid.

Excellent ethanol yield at high titer without detoxification in fermentation were achieved from both pretreatments, indicating the LSs produced from these two pretreatments are representative of biorefinery LSs. Separation of LS from the SPORL spent liquors were performed using an in-house pilot plant ultrafiltration (UF) system equipped with single-tube modules each with a separate permeate outlet [32]. The liquors were first centrifuged at $4000 \mathrm{rpm}$ for $20 \mathrm{~min}$ to remove solids. Two membranes ES404 and FP200 (Xylem PCI Membranes, Kostrzyn, Poland) that had cut-off molecular weight of 4 and $200 \mathrm{kDa}$, respectively, were used to remove low molecular weight impurities such as sugars and sugar
Table 4 The feedstock and pretreatment conditions used for the production of the two biorefinery LSs along with LS and ethanol yields

\begin{tabular}{|c|c|c|}
\hline & $\mathrm{Na}-\mathrm{LS}[31]$ & Ca-LS [32] \\
\hline Feedstock & Lodgepole pine wood & $\begin{array}{l}\text { Douglas-fir forest } \\
\text { residue }\end{array}$ \\
\hline \multicolumn{3}{|l|}{ Pretreatment conditions } \\
\hline T and time & $165^{\circ} \mathrm{C}$ for $60 \mathrm{~min}$ & $145^{\circ} \mathrm{C}$ for $4 \mathrm{~h}$ \\
\hline \multirow{2}{*}{$\begin{array}{l}\text { Chemical loadings } \\
\text { on wood }\end{array}$} & $2.2 \mathrm{wt} \% \mathrm{H}_{2} \mathrm{SO}_{4}$ & $2.4 \mathrm{wt} \%$ free $\mathrm{SO}_{2}$ \\
\hline & $8.0 \mathrm{wt} \% \mathrm{NaHSO}_{3}$ & $6.5 \mathrm{wt} \% \mathrm{Ca}\left(\mathrm{HSO}_{3}\right)_{2}$ \\
\hline Liquor to wood ratio & 3.00 & 3.55 \\
\hline $\begin{array}{l}\text { Fermentation total } \\
\text { solids }\end{array}$ & $20.0 \mathrm{wt} \%$ & $16.7 w t \%$ \\
\hline $\begin{array}{l}\text { Ethanol yield and } \\
\text { titer }\end{array}$ & 288 (L/tonne); $52.2 \mathrm{~g} / \mathrm{L}$ & 284 (L/tonne); $41.9 \mathrm{~g} / \mathrm{L}$ \\
\hline LS yield & $68 \mathrm{~kg} /$ tonne & $130 \mathrm{~kg} /$ tonne \\
\hline
\end{tabular}

degradation products (furans and organic acids, etc.) and very fine particular matters.

Naphthalene sulfonate formaldehyde condensate (FDN), a commercial dispersant for CWS from Zhanjiang additive company (Guangdong province, China), was used for comparison study.

Shenhua coal sample (Shenhua Coal CO., LTD., Datong, Shanxi, China) was crushed in a jaw crusher to obtain a small coal cake below $10 \mathrm{~mm}$ as described previously [6]. The crushed coal sample was dried under vacuum until reached at constant weight at $105{ }^{\circ} \mathrm{C}$. The dried coal was ball milled (Planetary ball mill QM-4F, Nanjing University Instrument Plant, Nanjing, China) for $2 \mathrm{~h}$ and screened using a 100 -mesh $(0.149 \mathrm{~mm}$ opening size) screen. The CWS as shown in Fig. 6 was prepared using the screen accept coal by continuously agitating the coal-water mixture at $60 \mathrm{wt} \%$ coal concentration at two dispersant concentrations of 0.75 and $1.0 \mathrm{wt} \%$. The mixture was continuously stirred for $10 \mathrm{~min}$ at $1200 \mathrm{rpm}$ to ensure homogenization.

The results of elemental and proximate analyses of the coal are listed in Table 5. The BET surface area of $12.10 \mathrm{~m}^{2} / \mathrm{g}$ and average particle size of $17.27 \mu \mathrm{m}$ were measured by nitrogen adsorption (ASAP2010, Micromeritics Instrument Corp., Norcross, USA) and dynamic light scattering (Mastersizer 2000, Malvern, Worcestershire, UK), respectively. The morphology of the coal powder was evaluated using SEM (Carl Zeiss AG EVO18, Oberkochen, Germany). The particle shape, the high inherent moisture, and the high oxygen content, suggested the coal was a low-rank metamorphic coal. This type of low quality coal is more difficult to prepare CWS with higher apparent viscosities compared with that of a high-rank coal [11]. 


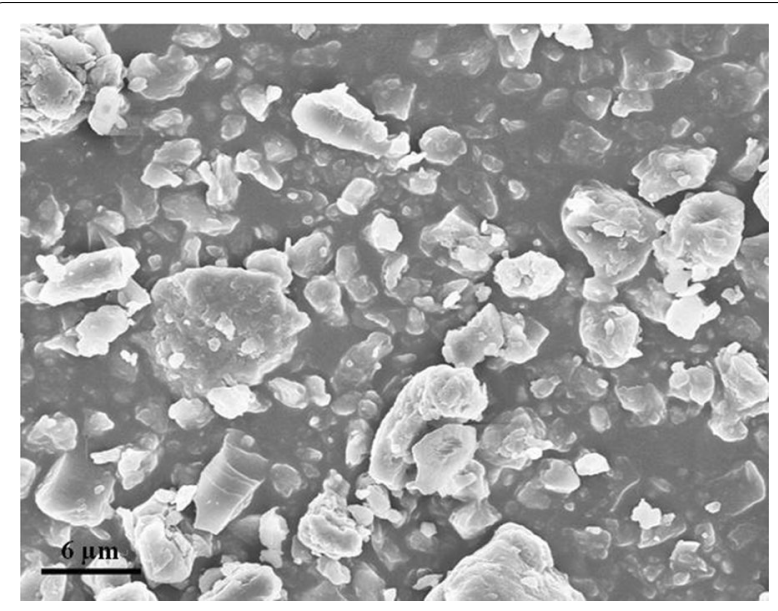

Fig. 6 A SEM image of the coal particles studied

Table 5 Proximate and ultimate analyses of the coal sample on air dried basis

\begin{tabular}{lr}
\hline Proximate analysis (wt\%) & \\
Inherent moisture & $7.23 \pm 0.11$ \\
Ash & $8.02 \pm 0.08$ \\
Volatile matter & $35.04 \pm 0.16$ \\
Ultimate analysis (wt\%) & \\
C & $81.35 \pm 0.13$ \\
H & $4.72 \pm 0.04$ \\
O & $11.66 \pm 0.08$ \\
N & $0.88 \pm 0.08$ \\
S & $0.51 \pm 0.04$ \\
\hline
\end{tabular}

\section{Fourier transform infrared and hydrogen nuclear magnetic} resonance spectra

The two LS samples were analyzed by Fourier transform infrared (FTIR) analysis using a Nicolet 380 FT-IR spectrometer (Thermo Scientific Nicolet, Waltham, MA, USA), as well as by hydrogen nuclear magnetic resonance $\left({ }^{1} \mathrm{H}-\mathrm{NMR}\right)$ spectroscopy using a Bruker DRX-500 spectrometer (Bruker Co., Ettlingen, Germany) at $25{ }^{\circ} \mathrm{C}$. Sample preparation for these analyses was described previously [33].

\section{LS molecular weight}

The molecular weight distributions of the dispersants were determined by aqueous gel-permeation chromatography (GPC) using Ultrahydrogel 120 and Ultrahydrogel 250 columns and UV detection at $280 \mathrm{~nm}$ (Waters 2487, Waters Co., MA, and USA). Sodium nitrate was used as mobile phase at a flow rate of $0.50 \mathrm{~mL} / \mathrm{min}$. Sodium polystyrene sulfonates with different molecular weights were used as standards for calibration. The uncertainty in calibration was less than $0.05 \%$.

\section{LS functional group contents}

The sulfonic acid and carboxyl groups content of LS were determined by non-aqueous conductometric titration $[18,34]$ using an automatic potentiometric titrator (809 Titrando, Metrohm Corp., Switzerland). The low molecular weight organic acids, inorganic salts, and other impurities were first removed by anion and cation exchange resins. Sodium hydroxide solution of $0.10 \mathrm{~mol} / \mathrm{L}$ was used as the titrant. Titration was conducted at $25^{\circ} \mathrm{C}$. The first-order peak of the titration curve were used to calculate sulfonic acid group content according to the following expression.

$$
S=\frac{C_{1-\mathrm{NaOH}} \cdot V_{1-\mathrm{NaOH}}}{m}
$$

where $S$ is sulfonic acid groups content $(\mathrm{mmol} / \mathrm{g}), C_{\mathrm{NaOH}}$ is the molar concentration of $\mathrm{NaOH}(\mathrm{mmol} / \mathrm{L}), V_{\mathrm{NaOH}}$ is the volume $(\mathrm{L})$ of $\mathrm{NaOH}$ solution used, $m$ is the mass of the LS sample (g). The $\mathrm{pH}$ change was 0.78 through titration.

The p-hydroxybenzoic acid was used as the internal standard and the tetrabutyl aqueous ammonia standard solution was used as the titrant to measure carboxyl group content [10].

Phenolic hydroxyl content was measured using FCreagent method [35]. Dried LS of $50 \mathrm{mg}$ was dissolved in $100 \mathrm{~mL}$ distilled water in a flask. An aliquot of $15 \mathrm{~mL}$ of the LS solution was mixed thoroughly with $1.5 \mathrm{~mL}$ of the FC-reagent and then added $5 \mathrm{~mL}$ of $20 \%(\mathrm{w} / \mathrm{v})$ $\mathrm{Na}_{2} \mathrm{CO}_{3}$ solution and adjusted the volume to $25 \mathrm{~mL}$ with distilled water. The mixture was kept stirring for $2 \mathrm{~h}$ at $30{ }^{\circ} \mathrm{C}$. Absorption measurements at $760 \mathrm{~nm}$ were carried out by a spectrophotometer (UV-2450, Shimadzu, Kyoto, Japan). Vanillin solutions were used for calibration.

\section{Determination of adsorption isotherms}

The amount of dispersants adsorbed onto CWS was measured by the residual mass fraction method. Firstly, dispersant solutions with different concentrations between 0.2 and $1.2 \mathrm{~g} / \mathrm{L}$ were added into CWS with coal powder consistency of $10 \mathrm{wt} \%$. Each mixture was mixed on a shaking bed at $200 \mathrm{rpm}$ for $5 \mathrm{~h}$ at $25^{\circ} \mathrm{C}$. The mixture was then centrifuged at $10,000 \mathrm{rpm}$ for $10 \mathrm{~min}$. The content of the dispersant in separated solution was measured by a UV spectrophotometer (UV2450, Shimadzu Corp., Tokyo, Japan) at $280 \mathrm{~nm}$. The amount of dispersant adsorbed was determined through calibration.

\section{Determination of Zeta potential of coal particles}

The zeta potential of coal particles was measured using a ZetaPALS analyzer (Brookhaven Instruments, 
Holtsville, NY, USA). Coal aqueous solutions of $0.2 \mathrm{wt} \%$ with different concentrations of dispersant were prepared. After shaking at $200 \mathrm{rpm}$ for $5 \mathrm{~h}$ at $25^{\circ} \mathrm{C}$, five replicate samples were taken and analyzed. The averages were reported.

\section{CWS rheological property}

The prepared CWS was allowed to stand for $5 \mathrm{~min}$. Measurements of rheological properties were performed by a rotational rheometer (RV I, Haake Corp., Karlsruhe, Germany) with a Z43 measure cup and a Z41 rotor at $25{ }^{\circ} \mathrm{C}$. The shear rate was first ramped up from 0 to $200 \mathrm{~s}^{-1}$ in $3 \mathrm{~min}$ and then ramped down in $3 \mathrm{~min}$. All measurements were taken at a shear rate of $100 \mathrm{~s}^{-1}$ during ramping up period. The measured viscosity value was the apparent viscosity.

\section{Authors' contributions}

FG and JYZ conducted pretreatments for lignosulfonates. YQ purified the lignosulfonates and drafted the manuscript. XL conducted viscosity measurements. WX conducted FTIR measurements. DY and JYZ designed study plan and edited manuscripts. DY conducted NMR measurements. All authors read and approved the final manuscript.

\section{Author details}

${ }^{1}$ School of Chemistry and Chemical Engineering, South China University of Technology, Guangzhou, China. ${ }^{2}$ USDA Forest Service, Forest Products Laboratory, Madison, WI, USA. ${ }^{3}$ School of Chemistry and Chemical Engineering, Yancheng Institute Technology, Yancheng, China.

\section{Acknowledgements}

This work was supported by a USDA Small Business Innovative Research (SBIR) Phase II project (Contract Number: 2010-33610-21589) to Biopulping International, a USDA National Institute of Food and Agriculture (NIFA) competitive Grant (No. 2011-68005-30416) through the Northwest Advanced Renewables Alliance (NARA), the International Science and Technology Cooperation Program of China (ISTCP): 2013DFA41670, the National Natural Science Foundation of China 21436004, and the Chinese Scholarship Council. The funding from these programs made the visiting appointments of Qin and Gu at the USDA Forest Products Laboratory (FPL) possible.

This work is conducted on official government time of Zhu while Qin and Gu are visiting scientists at the USDA Forest Products Lab.

\section{Competing interests}

Zhu is a co-inventor the SPORL process.

Received: 27 October 2015 Accepted: 11 May 2016

Published online: 02 June 2016

\section{References}

1. Bozell JJ, Petersen GR (2010) Technology development for the production of biobased products from biorefinery carbohydrates - the US Department of Energy's "top 10" revisited. Green Chem 12:539-554

2. Zhu JY, Zhuang XS (2012) Conceptual net energy output for biofuel production from lignocellulosic biomass through biorefining. Prog Energy Combust Sci 38:583-589

3. Zhu JY, Pan XJ (2010) Woody biomass pretreatment for cellulosic ethanol production: technology and energy consumption evaluation. Bioresour Technol 101:4992-5002

4. Ma R, Xu Y, Zhang X (2015) Catalytic oxidation of biorefinery lignin to value-added chemicals to support sustainable biofuel production. ChemSusChem 8:24-51
5. Zhu JY, Pan XJ, Wang GS, Gleisner R (2009) Sulfite pretreatment (SPORL) for robust enzymatic saccharification of spruce and red pine. Bioresour Technol 100:2411-2418

6. Zhou MS, Kong Q, Pan B, Qiu XQ, Yang DJ, Lou HM (2010) Evaluation of treated black liquor used as dispersant of concentrated coal-water slurry. Fuel 89:716-723

7. Phulkerd P, Thongchul N, Bunyakiat K, Petsom A (2014) Coal water slurry using dispersant synthesized from cashew nut shell liquid (CNSL). Fuel Process Technol 119:256-262

8. Miller BG, Miller SF, Morrison JL, Scaroni AW (1997) Cofiring coal-water slurry fuel with pulverized coal as a NOx reduction strategy. In: 14th annual international Pittsburgh coal conference, Taiyuan, Shanxi, 23-27 Sep 1997

9. Pisupati SV, Zarnescu V (2000) NOx reduction in pulverized coal combustors using waste coal as coal-water slurry. ACS Div Fuel Chem Prepr 45:499-503

10. Yang D, Qiu X, Zhou M, Lou H (2007) Properties of sodium lignosulfonate as dispersant of coal water slurry. Energy Convers Manag 48:2433-2438

11. Atesok G, Boylu F, Sirkeci AA, Dinçer H (2002) The effect of coal properties on the viscosity of coal-water slurries. Fuel 81:1855-1858

12. Li R, Yang DJ, Lou HM, Zhou MS, Qiu XQ (2012) Influence of sulfonated acetone-formaldehyde condensation used as dispersant on low rank coal-water slurry. Energy Convers Manag 64:139-144

13. Kakui T, Kamiya H (2004) Effect of sodium aromatic sulfonate group in anionic polymer dispersant on the viscosity of coal-water mixtures. Energy Fuels 18:652-658

14. Qiu XQ, Zhou MS, Yang DJ, Lou HM, Ouyang XP, Pang YX (2007) Evaluation of sulphonated acetone-formaldehyde (SAF) used in coal water slurries prepared from different coals. Fuel 86:1439-1445

15. Xu R, Zhuang W, He Q, Cai J, Hu B, Shen J (2009) Effects of chemical structure on the properties of carboxylate-type copolymer dispersant for coal-water slurry. AIChE J 55:2461-2467

16. Atesok G, Dincer H, Ozer M, Mütevellioğlu A (2005) The effects of dispersants (PSS-NSF) used in coal-water slurries on the grindability of coals of different structures. Fuel 84:801-808

17. Mishra S, Kanungo S (2003) Adsorption of sodium dodecyl benzenesulfonate onto coal. J Colloid Interface Sci 267:42-48

18. Qin Y, Yang D, Guo W, Qiu X (2015) Investigation of grafted sulfonated alkali lignin polymer as dispersant in coal-water slurry. J Ind Eng Chem 27:192-200

19. Zhou MS, Qiu XQ, Yang DJ, Lou HM, Ouyang XP (2007) High-performance dispersant of coal-water slurry synthesized from wheat straw alkali lignin. Fuel Process Technol 88:375-382

20. Abu-Dalo MA, Al-Rawashdeh NA, Ababneh A (2013) Evaluating the performance of sulfonated Kraft lignin agent as corrosion inhibitor for ironbased materials in water distribution systems. Desalination 313:105-114

21. Faix O (1992) Fourier transform infrared spectroscopy. In: Lin SY, Dence CW (eds) Book of methods in lignin chemistry. Springer-Verlag, Berlin

22. Da Silva LG, Ruggiero R, Gontijo PDM, Pinto RB, Royer B, Lima EC, Fernandes TH, Calvete T (2011) Adsorption of Brilliant Red 2BE dye from water solutions by a chemically modified sugarcane bagasse lignin. Chem Eng J 168:620-628

23. Tejado A, Pena C, Labidi J, Echeverria JM, Mondragon I (2007) Physicochemical characterization of lignins from different sources for use in phenol-formaldehyde resin synthesis. Bioresour Technol 98:1655-1663

24. Lundquist K, Stern K (1989) Analysis of lignins by 1 H NMR spectroscopy. Nord Pulp Pap Res J 4:210-213

25. Akiyama T, Matsumoto Y, Okuyama T, Meshitsuka G (2003) Ratio of erythro and threo forms of $\beta-\mathrm{O}-4$ structures in tension wood lignin. Phytochemistry 64:1157-1162

26. Lundquist K (1992) Proton (1H) NMR spectroscopy. Methods in lignin chemistry. Springer, Berlin, pp 242-249

27. Redlich O, Peterson DL (1024) A useful adsorption isotherm. J Phys Chem 1959:63

28. Foo KY, Hameed BH (2010) Insights into the modeling of adsorption isotherm systems. Chem Eng J 156:2-10

29. Pawlik M (2005) Polymeric dispersants for coal-water slurries. Colloids Surf Asp 266:82-90

30. Zhou M, Qiu X, Yang D, Wang W (2007) Synthesis and evaluation of sulphonated acetone-formaldehyde resin applied as dispersant of coalwater slurry. Energy Convers Manag 48:204-209 
31. Zhou H, Zhu JY, Gleisner R, Qiu X, Horn E, Negron J (2015) Pilot-scale demonstration of SPORL for bioconversion of lodgepole pine to bio-ethanol and lignosulfonate. Holzforschung. doi:10.1515/hf-2014-0332

32. Zhu JY, Chandra MS, Gu F, Gleisner R, Reiner R, Sessions J, Marrs G, Gao J, Anderson D (2015) Using sulfite chemistry for robust bioconversion of Douglas-fir forest residue to bioethanol at high titer and lignosulfonate: a pilot-scale evaluation. Bioresour Technol 179:390-397

33. Zhou H, Leu S-Y, Wu X, Zhu JY, Gleisner R, Yang D, Qiu X, Horn E (2014) Comparisons of high titer ethanol production and lignosulfonate properties by SPORL pretreatment of lodgepole pine at two temperatures. RSC Adv 4:27033-27038
34. Dence C (1992) Determination of carboxyl groups. Methods in lignin chemistry. Springer, Berlin, pp 458-464

35. de Sousa F, Reimann A, Björklund Jansson M, Nilberbrant N (2001)

Estimating the amount of phenolic hydroxyl groups in lignins. In: 11th ISWPC, Nice, France 2001, vol 3. pp 649-653

\section{Submit your manuscript to a SpringerOpen ${ }^{\circ}$ journal and benefit from:}

- Convenient online submission

\section{- Rigorous peer review}

- Immediate publication on acceptance

- Open access: articles freely available online

- High visibility within the field

- Retaining the copyright to your article 